\title{
EFECTOS DEL CRUZAMIENTO ENTRE LAS RAZAS DE CONEJOS NUEVA ZELANDA Y CALIFORNIA SOBRE CARACTERES DE LA CAMADA AL DESTETE
}

\section{EFFECTS OF CROSSBREEDING BETWEEN NEW ZEALAND AND CALIFORNIA RABBITS ON LITTER WEANING TRAITS}

\author{
Fernando Gallego ${ }^{1}$
}

${ }^{1}$ Zootecnista, Doctor en Ciencias Veterinarias, Docente Investigador Facultad de Ciencias Pecuarias. Universidad de Ciencias Aplicadas y Ambientales, U.D.C.A. Calle 222 No. 55-34, Bogotá, D.C., Colombia, e-mail: fgallego@ udca.edu.co

Rev. U.D.C.A Act. \& Div. Cient. 19(1): 115-121, Enero-Junio, 2016

\section{RESUMEN}

Con el objeto de evaluar la heterosis individual y materna y los efectos maternos sobre el tamaño de la camada, el peso y la sobrevivencia al destete, se establecieron cruces F1, entre las razas nueva Zelanda (NZ) y California (CAL). En una segunda etapa, las hembras cruzadas fueron apareadas a machos de la raza Azul de Viena (AV). Para estimar los efectos del grupo genético, la capacidad materna y la heterosis, se utilizaron modelos lineales generales. En F1, se encontraron diferencias en el tamaño de la camada y la sobrevivencia al destete entre cruces recíprocos, sin diferencias entre razas. La raza CAL, como madre en los cruces, presentó superioridad, al compararla con NZ. La heterosis individual en el peso al destete fue de $12,2 \%$, sobresaliendo el cruce $\mathrm{NZ} \times \mathrm{CAL}$, en el que también se complementó su mayor tamaño de camada, presentándose como el más productivo. El cruce terminal de hembras F1 apareadas a machos AV obtuvo mayor número de gazapos al destete, con relación a las hembras de razas puras; la heterosis materna fue de 2,78 y $1,46 \%$, para el peso y la sobrevivencia al destete, respectivamente. En definitiva, se recomienda el cruzamiento entre estas razas, utilizando a la raza California como base materna en F1, apareada en cruzamiento terminal a una tercera raza paterna, como la Azul de Viena, para mejorar la productividad de los sistemas de producción cunicola.

Palabras clave: Heterosis, efectos maternos, Oryctolagus cuniculus.

\section{SUMMARY}

In order to assess the presence of individual and maternal heterosis and maternal effects on litter size at birth and weaning weight and survival, under commercial conditions
F1 crosses between New Zealand and California breeds were established; in a second generation the females were mated to males of the Blue of Viena as terminal sire breed. For each genetic group six females, two males and their respective litters were used. As statistical procedures general linear models were implemented. As a result, in the progeny F1 significant differences in litter size and survival at weaning between reciprocal crosses were obtained; no differences between breeds were detected. The California breed used as maternal line presented superiority when compared to New Zealand females. The weaning weight variation was due to the number of young rabbits born and weaned per litter, maternal breed and genetic group. Also the results showed differences in maternal effects between breeds, which were favorables to California dams. The individual heterosis in weaning weight was 12.2 per cent for $\mathrm{NZ} \times \mathrm{CAL}$ genetic group. The terminal crossing of $\mathrm{F} 1$ females mated to Vienna Blue sires produced greater number of young rabbits and a more favorable weight at weaning relative to purebred females; maternal heterosis was 2.78 and 1.46 per cent for weight and weaning survival respectively. Finally for the improvement of rabbit production the use of dams NZ x CAL reared to AZ as terminal sire breed could be recommended.

Key words: Heterosis, maternal effects, Oryctolagus cuniculus.

\section{INTRODUCCIÓN}

La producción cunícola, se recomienda como una solución para los problemas nutricionales y económicos de los campesinos de los países en desarrollo (FAO, 2003). Asimismo, la demanda por la carne de conejo muestra condiciones para la expansión de la cunicultura, a nivel industrial y para la transferencia de tecnología hacia los sistemas de produc- 
ción, en la búsqueda de mayor eficiencia. En este sentido, las mejoras en los sistemas de alimentación y los programas de mejoramiento genético son las vías que ofrecen la posibilidad de incrementar los índices productivos, para hacer a esta actividad, productiva y rentable.

Nueva Zelanda y California son las razas más populares en la cunicultura mundial, reconocidas como razas maternas y paternas, respectivamente, por sus méritos en tamaño de la camada, habilidad lechera y alto crecimiento, con gran potencial de ser usadas en cruzamientos comerciales o para la formación de líneas, por sus habilidades combinatorias sobre otras razas (Blumetto, 2007).

El peso y el tamaño de la camada al destete son características que pueden resumir la eficiencia productiva de los sistemas de cría y determinar la rentabilidad de los sistemas de producción de conejos; estos caracteres están bajo la influencia de factores genéticos y ambientales (Blasco, 2002).

La selección de líneas ha sido la estrategia más importante en el mejoramiento genético de conejos para carne. La rentabilidad de los sistemas cunículas, se alcanza con programas de selección por prolificidad de las hembras (Savietto et al. 2014). Seleccionando líneas maternas por el número de gazapos al destete y vida útil reproductiva, Ragab et al. (2014), incrementaron el número de animales por hembra al sacrificio.

A su vez, Sorhue et al. (2014) encontraron altas y significativas correlaciones genéticas entre los rasgos productivos, medidos al nacimiento con los del destete. Estudios de Larzul \& Rochambeau (2005) mostraron que los animales seleccionados, para ganancia de peso, presentaron mejor eficiencia alimenticia, que los seleccionados por mayor tamaño de la camada; en los animales cruzados, la mayor ganancia de peso no estuvo asociada con incrementos en el número de gazapos, al nacimiento y destete

Actualmente, la producción intensiva se basa en esquemas de cruzamientos entre líneas; el más recomendado utiliza hembras F1, producto de líneas maternas, que son apareadas a una tercera raza; posteriormente, los conejos producidos se destinan al sacrificio (Antonini \& Cordiviola, 2010).

Vásquez et al. (2007), comparando los rendimientos de conejos Nueva Zelanda, Chinchilla y sus cruces, encontraron que para el peso, a los 35 días, no existieron diferencias entre los cruces recíprocos y Nueva Zelanda; en el estudio, no fue importante la vía del cruzamiento y la raza Chinchilla fue significativamente la de menor peso.

Los cruces terminales de machos de la raza Gigante de Flandes con hembras F1 fueron más pesados al sacrificio, que las razas puras y que otros cruces entre las razas $\mathrm{NZ}$ y CAL (García et al. 2000a). La raza utilizada como macho en cruzamientos no presentó efectos sobre los rasgos de la camada, medidos durante la lactancia y fue importante fuente de variación sobre la ganancia de peso y la calidad de la canal (Cheeke \& Patton, 2003).

Piles et al. (2005) encontraron superioridad en ganancia de peso, tasa de natalidad y tasa de destete, en los genotipos cruzados sobre el promedio de sus padres puros, como también la hallaron Ragab \& Baselga (2011).

La selección de líneas maternas, para la expresión de rasgos pre y al destete y de líneas paternas, para el crecimiento posdestete, se presenta como una alternativa favorable para lograr incrementos significativos en las principales características de la producción y en la rentabilidad del sistema (García et al. 2000b).

Matics et al. (2014) mostraron bajos efectos aditivos, cuando estimaron parámetros genéticos de caracteres reproductivos en líneas maternas de Nueva Zelanda, recomendando los cruzamientos entre líneas o razas sobresalientes en características maternas, con líneas de alto crecimiento o paternas, como sistema de mejoramiento, para incrementar la producción.

Zeferino et al. (2011) y Lukefahr et al. (2000) encontraron efectos ambientales sobre el peso al nacimiento y destete, debido al año, época y finca y temperatura, como efectos ambientales de los individuos a la edad, edad al primer parto e intervalo entre partos de la madre y al tamaño de la camada de la que provino el gazapo.

Ponce de León et al. (2002) hallaron efectos significativos de la raza sobre la viabilidad de la camada a la cuarta semana de edad, utilizando las razas California y Nueva Zelanda; además en sus resultados, no encontraron diferencias entre razas para sobrevivencia; en ganancia de peso, se presentaron diferencias significativas entre razas y entre tamaños de camada diferentes.

Cuando las razas y las líneas han sido seleccionadas por rasgos relacionados con el tamaño y el peso de la camada al destete, la media de las F1 dialélicas no presenta heterosis positiva; la heterosis estimada para los caracteres de crecimiento, en experimentos de cruzamiento, suele ser baja, entre el 3 y 10\% (García et al. 2008).

La estimación de la heterosis para los cruzamientos entre las líneas seleccionadas fue alrededor del 6,4\%, para el número de nacidos totales y del $7,4 \%$, para el número de nacidos vivos (García et al. 2000b). 
Brun \& Baselga (2005) calcularon heterosis del 15,2, 20,1 y $6,6 \%$, para el número de nacidos totales, vivos y destetados, respectivamente, para cruces a partir de líneas maternas.

Las heterosis estimadas para el total de nacidos y nacidos vivos fueron significativas y variaron entre $-4,6$ y $-6,5 \%$; los efectos paternos fueron favorables a las razas nueva Zelanda y California, debido a las diferencias entre cruces. Nueva Zelanda sobresalió sobre las otras razas y cruces (García et al. 2013).

En la producción cunícula, los cruzamientos más utilizados son a partir de dos razas para producir híbridos F1 (Baselga, 2004); los cruces recíprocos permitirían estimar la influencia de cada uno sobre la variación en fertilidad y en tamaño de la camada; en estos casos, la generación F1 supera los valores de sus parentales.

Jakubec \& Hyanek (1982) definen a un súper carácter como la complementariedad que se obtiene en los cruzamientos o la ventaja de un cruce sobre otros o sobre una raza pura, en la forma en la que en él se combinan dos o más características y cómo se complementan entre ellas; los súper caracteres deben ser mensurables y expresados como unidades de producción. Asumiendo una composición multiplicativa de sub caracteres en un carácter y una combinación lineal de caracteres en un súper carácter:

$$
\mathrm{Z}=(\mathrm{X} 1 \times \mathrm{X} 2)(\mathrm{X} 2-1)
$$

Dónde: $\mathrm{Z}=$ media del supercaracter; $\mathrm{X} 1=$ Promedio de las gazapos al nacimiento por hembra y por grupo genético; $\mathrm{X} 2$ =Porcentaje de sobrevivencia al destete; X 2-1 = Media del peso al destete.

El objetivo de esta investigación fue evaluar los efectos de los cruzamientos entre las razas Nueva Zelanda y California sobre el tamaño y el peso de la camada al destete, estimando efectos maternos, heterosis individual y heterosis materna.

\section{MATERIALES Y MÉTODOS}

La investigación, se realizó en la Universidad de Ciencias Aplicadas y Ambientales U.D.C.A, localizada en Bogotá, D.C., a $2.640 \mathrm{msnm}$ y con temperatura mínima de $4^{\circ} \mathrm{C}$ y máxima de $17^{\circ} \mathrm{C}$. El estudio, se desarrolló en condiciones comerciales estándar, utilizando jaulas metálicas y de parto con nidal subpiso. Los animales recibieron alimento balanceado comercial, de acuerdo a la etapa productiva en que se encontraban y agua a voluntad.

Como reproductores, para todos los apareamientos, se utilizaron seis hembras de primer parto y dos machos, estableciendo los cuatro siguientes grupos genéticos: 1) Nueva
Zelanda (NZ); 2) California (CAL) y sus cruces; 3) NZ x CAL y 4) CAL x NZ.

En una segunda etapa, las hembras cruzadas fueron apareadas con machos de la raza Azul de Viena, generando los siguientes grupos genéticos: 1) AZ x (CAL x NZ); 2) AZ x (NZ x CAL); 3) AZ x (CAL x CAL) y 4) AZ x (NZ x NZ).

A los machos, previamente se les realizó un espermiograma, con el fin de reducir la variación individual en el tamaño de la camada. Las hembras fueron sincronizadas hormonalmente, para inducir los partos para el mismo periodo. La duración del periodo de lactancia fue de 28 días, se hizo el destete de todas las camadas a edad fija.

Análisis de la información: Se utilizaron los análisis de varianza en el paquete estadístico SAS, bajo procedimientos lineales generales. Las variables analizadas fueron: sobrevivencia al destete, tamaño y peso de la camada al nacimiento y al destete. Se utilizó un diseño completamente al azar, con 6 repeticiones por tratamiento o grupo genético, que correspondió a una hembra y a su camada, de acuerdo al siguiente modelo: $Y i K l=\alpha \mathbf{i}+\beta \mathbf{j}+(\alpha \beta) i \mathbf{j}+\rho \mathrm{K}+\mathrm{e} \mathbf{i j k}$

Dónde:

$\alpha \mathrm{i}=$ efecto de la i-ésima raza de la hembra en su progenie. $\beta \mathrm{j}=$ efecto de la j-ésima raza del macho sobre su progenie. $(\alpha \beta)$ ij = efecto de la interacción de la i-ésima línea materna con la j-ésima línea paterna.

$\rho K=$ efecto del tamaño de la camada sobre el peso.

e i j k l = error experimental, distribuido normalmente $\left(0, \sigma^{2}\right.$ e).

La sobrevivencia fue estimada como la diferencia en porcentaje entre el número de nacidos vivos y el número de gazapos destetados por camada.

Efectos genéticos: Modelo dialélico de los cruzamientos para estimar: heterosis individual y efectos maternos o capacidad materna, de acuerdo al siguiente modelo:

$$
X i j=\mu+1 / 2 l i+1 / 2 l j+m j+h i j ;
$$

Dónde: $\mu=$ media de las líneas parentales; li $=$ efecto aditivo directo de la línea i; lj = efecto aditivo directo de la línea j; mi $=$ efecto aditivo materno línea j; $\mathrm{hij}=$ heterosis del cruce $\mathrm{i} \mathrm{x} \mathrm{j}$.

Los efectos maternos o capacidad materna $(\mathrm{m})$ fueron calculados como la diferencia entre cruces cuando la raza i interviene como madre menos el desempeño materno de la raza $\mathrm{j}$

$$
\mathrm{m}=\mu \mathrm{lj}-\mu \mathrm{li}
$$


La heterosis individual $(\mathrm{H})$ fue estimada como:

\% $\mathrm{H}=\mu \mathrm{F} 1-\mu \mathrm{P} / \mu \mathrm{P} \times 100$ (Falconer \& Mackay, 1996; Ruales et al. 2007).

Para la segunda generación, la heterosis materna $(\mathrm{Hm})$ se estimó como:

$\% \mathrm{Hm}=\mu(\mathrm{F} 1 \times \mathrm{AZ})-\mu(\mathrm{P} \times \mathrm{AZ}) / \mu(\mathrm{P} \times \mathrm{AZ}) \times 100$ (Campos, 2004).

Dónde: F1, es el promedio de las cruzadas y P, es el promedio de las razas parentales puras.

\section{RESULTADOS Y DISCUSIÓN}

Tamaño y sobrevivencia de la camada durante la cría: El modelo estadístico encontró, como fuentes significativas de variación, a los efectos paternos, maternos, grupo genético y tamaño de la camada; estos resultados concuerdan con los estudios realizados por Piles et al. (2005).

El promedio de gazapos por hembra y por parto fue de 8,46 $\pm 1,8$, que fue superior al descrito por Vásquez et al. (2007), en la raza Nueva Zelanda, en la Sabana de Bogotá.
Se encontraron diferencias significativas en el tamaño de la camada y la sobrevivencia al destete; debido al grupo genético (Tabla1) fueron superiores y sin diferencias entre ellos, las camadas de todos los grupos sobre el cruce de CAL $x$ NZ, con promedio de 7,33 \pm 15 gazapos por hembra. No se encontraron interacciones entre las variables y tampoco diferencias entre las medias de las razas parentales y sus cruces en la sobrevivencia al destete. Entre grupos, se presentaron diferencias entre la raza California y el cruce NZ x CAL. Lukefahr et al. (2000) tampoco encontraron efectos del cruzamiento entre las razas Altex y NZ, sobre el tamaño de la camada al nacimiento y al destete.

Peso al destete: Presentó un promedio de 605,6 \pm 61,7g, que es similar con lo registrado por Vásquez et al. (2007), en gazapos de las razas Chinchilla y Nueva Zelanda, en condiciones ambientales y de manejo muy similares a las de esta investigación. Asimismo, concuerda con el promedio descrito por Ponce de León et al. (2002).

El análisis estadístico evidenció, como fuentes significativas de variación en el peso al destete, a los efectos de la raza materna y paterna, como también al grupo racial y al tamaño de la camada al destete (Tabla 1).

Tabla 1. Promedios del número de nacidos, sobrevivencia y peso al destete, en cruces terminales de tres razas.

\begin{tabular}{|l|c|c|c|}
\hline \multicolumn{1}{|c|}{ Composición Racial } & Número de Nacidos & Sobrevivencia (\%) & Peso Destete (g) \\
\hline CAL x CAL & $9,25^{\mathrm{a}}$ & $94,4^{\mathrm{a}}$ & $569,3^{\mathrm{c}}$ \\
\hline NZ x NZ & $8,75^{\mathrm{a}}$ & $91,8^{\mathrm{a}}$ & $653,7^{\mathrm{b}}$ \\
\hline CAL x NZ & $7,33^{\mathrm{b}}$ & $94,8^{\mathrm{a}}$ & $654,7^{\mathrm{b}}$ \\
\hline NZ x CAL & $9,10^{\mathrm{a}}$ & $89,7^{\mathrm{b}}$ & $717,5^{\mathrm{a}}$ \\
\hline
\end{tabular}

Promedios con diferente letra en el superíndice presentan diferencias $(P<00.5)$.

Los gazapos con padres de la raza NZ alcanzaron, significativamente, mayor peso que las progenies, cuyo padre fue de la raza CAL, producto de los efectos aditivos de los machos NZ y de la heterosis individual en este carácter y de efectos maternos de la raza CAL, resultados también descritos por Larzul \& Rochambeau (2005).

El número de gazapos nacidos vivos por camada afectó, significativamente, al peso individual al destete; se encontraron diferencias entre las camadas de 7 gazapos al nacimiento, que fueron las de mayor peso al destete, con relación a camadas de otros tamaños que, en este trabajo, estuvieron dis- tribuidas en el rango de 5 a 11 gazapos al nacimiento, efecto también encontrado por García et al. (2008).

Los gazapos de mayor peso fueron del cruce NZ x CAL, con promedio de $717,5 \mathrm{~g}$; entre cruces recíprocos, se encontraron diferencias que pueden ser por efectos maternos y, posiblemente, a la habilidad combinatoria específica del cruce. Las crías de la raza California fueron, significativamente, las más livianas, en comparación con los demás grupos genéticos evaluados. La heterosis en el peso al destete fue positiva y con un valor de $12,24 \%$. 
Las diferencias significativas entre los cruces recíprocos pueden ser atribuidas a los efectos maternos de las razas (Tabla 2). Las hembras Californianas presentaron el mayor número de gazapos nacidos y el mayor peso al final de la lactancia, como también se presentaron en el estudio de Lukefahr et al. (2000).
Las hembras de la raza California fueron superiores a las Nueva Zelanda, en rasgos considerados como principales, para los sistemas de cría, como son el número de gazapos que llegan al destete y el peso al destete. La capacidad materna de CAL fue de 0,88 gazapos por camada y de 57,3g al destete, sobre la raza NZ.

Tabla 2. Efecto materno sobre el número de nacidos y el número de gazapos y peso al destete.

\begin{tabular}{|l|c|c|c|}
\hline \multicolumn{1}{|c|}{ Raza Materna } & Número de Nacidos & $\begin{array}{c}\text { Sobrevivencia } \\
\mathbf{( \% )}\end{array}$ & $\begin{array}{c}\text { Peso Destete } \\
(\mathbf{g})\end{array}$ \\
\hline California & $9,16^{\mathrm{a}}$ & $92,1^{\mathrm{a}}$ & $713,56^{\mathrm{a}}$ \\
\hline Nueva Zelanda & $8,04^{\mathrm{b}}$ & $93,3^{\mathrm{a}}$ & $656,23^{\mathrm{b}}$ \\
\hline$\%$ Heterosis & $-0,088^{\mathrm{b}}$ & $-0,91$ & 12,2 \\
\hline
\end{tabular}

Promedios con diferente letra en el superíndice presentan diferencias $(P<00.5)$.

Al destete llegaron más gazapos vivos de los grupos CAL y CAL x NZ, con un número significativamente superior a los otros grupos; la heterosis individual del peso al destete en los cruces fue de $12,2 \%$, que se considera alta y de mayor impacto sobre la productividad en los sistemas de cría.

La heterosis encontrada para el total de nacidos vivos y su sobrevivencia durante la cría fue muy baja y negativa (Tabla 2), a diferencia de Brun \& Baselga (2005) y de García et al. (2000b), quienes encontraron efectos positivos ocasionados por la heterosis y valores mayores al $6 \%$ de superioridad de los individuos cruzados.

El cruce NZ x CAL presentó una superioridad en el peso al destete del $26 \%$ con relación a sus razas parentales que, complementado con su tamaño de camada al nacimiento, lo muestra como el más productivo de los grupos genéticos evaluados.
Desempeño de hembras F1: Las hembras cruzadas F1, se desempeñaron como mejores madres, que las hembras de razas puras, a las que superaron en el total de gazapos destetados y en sus respectivos pesos (Tabla 3). El número de nacidos por camada fue causa de variación significativa en el peso individual al nacimiento y destete; los mayores pesos correspondieron con las camadas comprendidas entre 6 y 9 gazapos al nacimiento.

En el análisis de varianza fueron causa significativa de variación sobre el peso al destete, el número de nacidos y destetados, el grupo genético y los efectos maternos. El promedio en el número de nacidos vivos de las hembras F1 apareadas a la raza Azul de Viena no presentó diferencias estadísticas, con el de las hembras puras.

Los valores positivos de heterosis hallados para tamaño de camada y sobrevivencia fueron menores a los registrados por

Tabla 3. Desempeño de hembras F1, expresado en promedios del número de nacidos, sobrevivencia y peso al destete.

\begin{tabular}{|l|c|c|c|}
\hline \multicolumn{1}{|c|}{ Composición Racial } & Número de Nacidos & Sobrevivencia (\%) & Peso Destete (g) \\
\hline AZ x (CAL x CAL) & $9,12^{\mathrm{a}}$ & $87,8^{\mathrm{b}}$ & $689,3^{\mathrm{b}}$ \\
\hline AZ x (NZ x NZ) & $8,5^{\mathrm{b}}$ & $90,4^{\mathrm{ab}}$ & $665,7^{\mathrm{b}}$ \\
\hline AZ x(CAL $\times$ NZ) & $8,3^{\mathrm{b}}$ & $92,2^{\mathrm{a}}$ & $715,7^{\mathrm{a}}$ \\
\hline AZ x (NZ x CAL) & $9,6^{\mathrm{a}}$ & $94,6^{\mathrm{a}}$ & $671,5^{\mathrm{b}}$ \\
\hline Heterosis Materna & $1,7^{\mathrm{b}}$ & 4,82 & 2,78 \\
\hline
\end{tabular}

Promedios con diferente letra en el superíndice presentan diferencias $(P<00.5)$ 
Brun \& Baselga (2005), para hembras de líneas artificiales y similares a los estimados por García et al. (2013), en hembras cruzadas, de las razas presentes en esta investigación.

Las variables de sobrevivencia y de peso al destete en los gazapos de hembras cruzadas $\mathrm{F} 1$ fueron significativas, con heterosis maternas de 4,82 y $2,78 \%$, respectivamente (Tabla 3 ). El cruce de machos Azul de Viena en hembras NZ x CAL mostró ventajas sobre los demás cruces y efectos de complementación, que se presentaron entre las variables número de nacidos y sobrevivencia, con promedio de 9,1 gazapos al destete y aun cuando el peso promedio al destete de este cruce fue menor a otros cruces (Tabla 3), como súper carácter produce 6,12 kilogramos de gazapos en pie por hembra, que lo hacen más productivo, que los otros cruces estudiados.

Agradecimientos: Esta investigación fue financiada por la Universidad de Ciencias Aplicadas y Ambientales U.D.C.A. Conflicto de intereses: El manuscrito fue preparado y revisado por el autor quien declara que no existe ningún conflicto de intereses que ponga en riesgo la validez de los resultados presentados.

\section{BIBLIOGRAFÍA}

1. ANTONINI, A.G.; CORDIVIOLA, C. 2010. Mejoramiento genético en conejos para carne. J. Basic Appl. Genetics. 21:2-7.

2. BASELGA, M. 2004. Genetic improvement of meat rabbits. Programs and diffusion. Proc. 8th World Rabbit Congress, Puebla México.13p.

3. BLASCO, A. 2002. La mejora genética del conejo en España en los últimos 25 años. XXVII Simposium de Cunicultura, 21-24 Mayo 2002. Reus (España). 21p.

4. BLUMETTO, O. 2007 Guía para el manejo de líneas genéticas de alto potencial en conejos para carne. Ed .Hemisferio Sur. 124p.

5. BRUN, J.M.; BASELGA, M. 2005. Analysis of reproductive performances during the formation of a rabbit synthetic strain. World Rabbit Sci.13:237-250.

6. CAMPOS, J. 2004. Melhoramiento genético aplicado â produçao animal. FEPMVZ. Belo Horizonte. 609p.

7. CHEEKE, P.R.; PATTON, M. 2003. Potentials of rabbit production in tropical and subtropical agricultural systems. J. Anim. Sc. 63:1581-1586.
8. FALCONER, D.S.; MACKAY, T.F.C. 1996. Introduction to quantitative genetics. $4^{\text {th }}$ Edition, Prentice Hall, Longman Group Limited. 469p.

9. FAO. 2003. World agriculture towards 2015/2030. An FAO perspective. Ed. J. Brunsima. London. 511p.

10. GARCÍA, Y.; PONCE DE LEÓN, R.; GUZMÁN, G. 2008. Fuentes genéticas de variación que influyen en la fertilidad y en rasgos del nacimiento. Rev. Cubana Cienc. Agr. 42(4):341-345.

11. GARCÍA, Y.; PONCE DE LEÓN, R.; GUZMÁN, G. 2013. Efectos aditivos directos maternos y heterosis en rasgos de prolificidad al nacer en conejos. Rev. Cubana Cienc. Agr. 47(4):335-340.

12. GARCÍA, M.L.; BASELGA, M.; LAVARA, R.; LAVARA, F.; VICENTE, J.S. 2000a. Reproductive characters in crossbreeding among three maternal lines of rabbits. VII .World Rabbit Congress, A. Julio 2000. Valencia. España. p.397-402.

13. GARCÍA, M.L.; BASELGA, M.; VICENTE, J.S.; LAVARA, R. 2000b. Selection response on reproductive characters in a maternal line of rabbits. VII World Rabbit Congress, A: Julio 2000. Valencia. España. p.381-387.

14. JAKUBEC, V.; HYANEK. J. 1982. Quantitative analysis of components of hybridization. Livestock Prod. Sc. 9:639-351.

15. LARZUL, C.; ROCHAMBEAU, H. 2005. Selection for residual feed consumption in the rabbit. Livestock Prod. Sc. 95:67-72.

16. LUKEFAHR, S.D.; NKWOCHA, H.I.; NJAKOH, H.; TAWAH, E.; AKOB, J.M.; KONGYU, F.A.; NJWE, R.M.; GUDAHL. D. 2000. Present status of the Heifer Project International - Cameroon Rabbit Programme: Back to the future. World Rabbit Sci. 8:75-83.

17. MATICS, Z.S.; NAGY, I.; GERENCSÉR, Z.S.; RADNAI, I.; GYOVAI, P.; DONKÓ, T. 2014. Pannon breeding program in rabbit at Kaposvár University. World Rabbit Sci. 22(4):287-300.

18. PILES, M.; RAFEL, O.; RAMON, J.; VARONA, L. 2005. Genetic parameters of fertility in two lines of rabbits with different reproductive potential. J. Anim. Sci. 83:340-343. 
19. PONCE DE LEÓN, R.; GUZMÁN, G.; QUESADA, M. 2002. Crecimiento y Eficiencia Alimentaria de cuatro razas de conejos. Rev. Cubana Cienc. Agr. 36(1):7-14

20. RAGAB, M.; BASELGA, M. 2011. A comparison of reproductive traits of four maternal lines of rabbits selected for litter size at weaning and founded on different criteria. Livestock Sci. 136(2):201-206.

21. RAGAB, M.; SÁNCHEZ, J.P.; MÍNGUEZ, C.; VICENTE, J.S.; BASELGA, M. 2014. Litter size components in a full diallel cross of four maternal lines of rabbits1. J. Animal Sci. 92(8):1-6.

22. RUALES, F.R.; MANRIQUE, C.; CERÓN, M. 2007. Fundamentos en mejora genética animal. Ed. Medellín Colombia: L. Vieco e hijas Editores. 208p.

23. SAS/STAT. 2007. Manual of users guide statistics. Version 6. SAS Institute Inc. Cary, NC, U.S.A.

24. SAVIETTO, D.; CERVERA, C.; RÓDENAS, L.; MARTÍNEZ, P.E.; BASELGA, M.; GARCÍA, D.F. 2014. Different resource allocation strategies result from selection for litter size at weaning in rabbits. Int. J. Animal Biosci. 8(4):618-628.

25. SORHUE, U.G.; MMEREOLE, F.U.C.; IRIKEFE-EKEKE, E.P.; NWAKWO, S.U. 2014.Genetic analysis of some economic traits in a composite breed of domestic rabbits reared in a tropical environment. Nigerian J. Genetics. 28(2):34-37.

26. VÁSQUEZ, R.; MARTIINEZ, R.; MANRIQUE, C.; RODRÍGUEZ, Y. 2007. Evaluación genética del comportamiento productivo y reproductivo en núcleos de Conejos de las razas Nueva Zelanda y Chinchilla. Rev. Corpoica, Ciencia y Tecnología Agropecuaria. 8(1):69-74.

27. ZEFERINO, C.P.; MOURA, A.S.; FERNANDES, S.; KANAYAMA, J.S.; SCAPINELLO, C.; SARTORI, J.R. 2011.Genetic group $x$ ambient temperature interaction effects on physiological responses and growth performance of rabbits. Livestock Sc. 140:177-183.

Recibido: Noviembre 9 de 2015

Aceptado: Mayo 19 de 2016

Cómo citar.

Gallego, F. 2016. Efectos del cruzamiento entre las razas de conejos Nueva Zelanda y California sobre caracteres de la camada al destete. Rev. U.D.C.A Act. \& Div. Cient. 19(1): 115-121. 